\title{
Improved Basic Block Reordering
}

\author{
Andy Newell and Sergey Pupyrev
}

\begin{abstract}
Basic block reordering is an important step for profile-guided binary optimization. The state-of-the-art goal for basic block reordering is to maximize the number of fall-through branches. However, we demonstrate that such orderings may impose suboptimal performance on instruction and I-TLB caches. We propose a new algorithm that relies on a model combining the effects of fall-through and caching behavior. As details of modern processor caching is quite complex and often unknown, we show how to use machine learning in selecting parameters that best trade off different caching effects to maximize binary performance.

An extensive evaluation on a variety of applications, including Facebook production workloads, the open-source compilers Clang and GCC, and SPEC CPU benchmarks, indicate that the new method outperforms existing block reordering techniques, improving the resulting performance of applications with large code size. We have open sourced the code of the new algorithm as a part of a post-link binary optimization tool, BOLT.
\end{abstract}

Index Terms-Code generation, Code layout, Optimizing compilers, Profile-guided optimizations, Graph algorithms

\section{INTRODUCTION}

$\mathrm{P}$ ROFILE-guided binary optimization (PGO) is an important step for improving performance of large-scale applications that tend to contain huge amounts of code. Such techniques, also known as feedback-driven optimization (FDO), are designed to improve code locality which leads to better utilization of CPU instruction caches. In practice tools like AutoFDO [1], Ispike [2], PLTO [3], HFSort [4], and BOLT [5] speed up binaries by $5 \%-15 \%$ depending on workload and CPU architecture, and thus, are widely used for a variety of complex applications.

PGO is comprised of a number of optimization passes such as function and basic block reordering, identical code folding, function inlining, unreachable code elimination, register allocation, and others. Typical targets for optimizations are an instruction cache (I-cache) used to hold executable instructions and a translation lookaside buffer (ITLB) used to speed up virtual-to-physical address translation for instructions. The reordering passes directly optimize code layout, and thus impact performance the most [2], [5]. Therefore, even small improvements in the underlying algorithms for code reordering significantly affect the benefit of PGO tools.

Current techniques for basic block reordering optimize a specific dimension of CPU performance such as (i) cache line utilization by increasing the average number of instructions executed per cache line, (ii) the branch predictor by reducing the number of mispredicted branches, and (iii) the instruction cache miss rate by minimizing cache line conflicts. An application's overall performance depends on a combination of these dimensions. Since modern processors employ a complex and often non-disclosed strategy for execution, it is challenging to consider all of these effects at once when optimizing an ordering of basic blocks. In this paper, we make the first, to the best of our knowledge, attempt to design and implement a block reordering algorithm that directly optimizes

- A. Newell is with Facebook, Inc., Menlo Park, CA, USA. E-mail: newella@fb.com

- S. Pupyrev is with Facebook, Inc., Menlo Park, CA, USA.

E-mail: spupyrev@fb.com the performance of an application.

Our approach consists of two main steps. Firstly, we learn a proxy metric that describes the relationship between the performance of a binary and the ordering of its basic blocks. This is achieved by (i) identifying a set of features representing how basic block ordering can influence performance, (ii) collecting training data by running extensive experiments and measuring the performance, and (iii) using machine learning to select the best combination of the features for a score that best predicts CPU performance. Secondly, we suggest an efficient algorithm that, given a control flow graph for a procedure, builds an improved ordering of the basic blocks optimizing the learned metric. Since the constructed metric correlates highly with the performance of a binary, we observe overall efficiency gains, despite possible regressions of individual CPU characteristics.

The contributions of the paper are the following.

- We identify an opportunity for improvement over the classical approach for basic block reordering, initiated by Pettis and Hansen [6]. Then we extend the model and suggest a new optimization problem with the objective closely related to the performance of a binary.

- We then develop a new practical algorithm for basic block reordering. The algorithm relies on a greedy technique for solving the optimization problem. We describe the details of our implementation, which scales to real-world instances without significant impact on the running time of a binary optimization tool.

- We propose a Mixed Integer Programming formulation for the aforementioned optimization problem, which is capable of finding optimal solutions on small functions. Our experiments with the exact method demonstrate that the new suggested heuristic finds an optimal ordering of basic blocks in $98 \%$ of real-world functions with 30 or fewer blocks.

- Finally, we extensively evaluate the new algorithm on a variety of applications, including Facebook production workloads, open-source compilers, Clang and GCC, and SPEC CPU 2017 benchmarks. The experiments indicate that the new method outperforms the state-of-the-art 
block reordering techniques, improving the resulting performance by $0.5 \%-1 \%$. We have open sourced the code of our new algorithm as a part of BOLT [5], |7].

The paper is organized as follows. We first discuss limitations of the existing model for basic blocks reordering and suggest an improvement in Section 2 We describe an efficient heuristic (Section 3) and an exact algorithm (Section 4) for solving the new problem. Next, in Section 5 we present experimental results, which are followed by a discussion of related work in Section 6 We conclude the paper and discuss possible future directions in Section 7

\section{Learning an Optimization Model}

The state-of-the-art approach for basic block reordering is based on the idea of collocating frequently executed blocks together. The goal is to position blocks so that the hottest successor of a block will most likely be a fall-through branch, that is, located right next to the predecessor. This strategy reduces the number of taken branches and the working set size of the I-cache, while relieving pressure from the branch predictor unit. More formally, the reordering problem can be formulated as follows. Given a directed control flow graph comprising of basic blocks and frequencies of jumps between the blocks, find an ordering of the blocks such that the number of fall-through jumps is maximized. This is the maximum directed TRAVELING SALESMAN PROBLEM (TSP), a widely studied NP-hard combinatorial optimization problem.

The simplicity of the model and solid practical results made TSP-based algorithms very popular in the code optimization community. To the best of our knowledge, Boesch and Gimpel $[8]$ are the first ones to formulate the problem of finding an ordering of basic block as the path covering problem on a control flow graph, which is equivalent to solving TSP. They describe an optimal algorithm on acyclic directed graphs and suggest a heuristic for general digraphs. Later the same path covering model has been studied in a series of papers suggesting optimal algorithms for special classes of digraphs and heuristics for general digraphs [9], [10]. In their seminal paper from 1990 [6], Pettis and Hansen present two heuristics for positioning of basic blocks. We observe that both heuristics are designed to solve (possibly non-optimally) TSP. Later, one of the heuristics (seemingly producing better results) has been extended by Calder and Grunwald [11], Torrellas et al. [12], and Luk et al. [2]. We stress that the majority of existing algorithms for block reordering utilize the TSP model. A variant of the PettisHansen algorithm is used by most of the modern binary optimizers, including PLTO [3], Ispike [2], BOLT [5], and the link-time optimizer (LTO) of the GCC compiler [13].

Notice that solving TSP alone is not sufficient for constructing a good ordering of basic blocks. It is easy to find examples of control flow graphs with multiple different orderings that are all optimal with respect to the TSP objective. Consider for example a control flow graph in Fig. 1 in which the maximum number of fall-through branches is achieved with two orderings that utilize a different number of I-cache lines in a typical execution. For these cases, an algorithm needs to take into consideration non-fall-through branches to choose the best ordering. However, maximizing
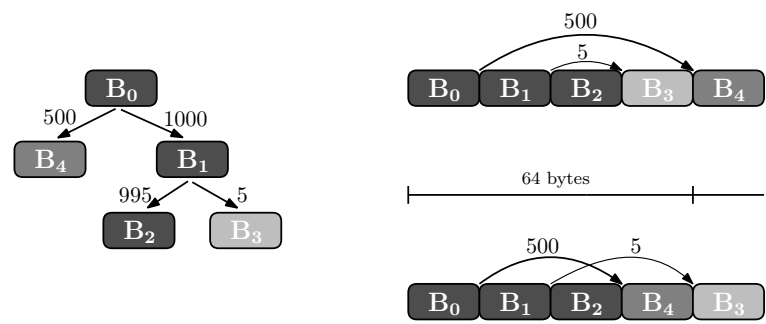

Fig. 1. Two orderings of basic blocks with the same TSP score (1995) resulting in different I-cache utilization. All blocks have the same size of 16 bytes and colored according to their hotness in the profile.

the number of fall-through jumps is not always preferred from the performance point of view. Consider a control flow graph with seven basic blocks in Fig. 2 It is not hard to verify that the ordering with the maximum number of fall-through branches is one containing two concatenated chains, $B_{0} \rightarrow B_{1} \rightarrow B_{3} \rightarrow B_{4}$ and $B_{5} \rightarrow B_{6} \rightarrow B_{2}$ (upper-right in Fig. 2). Observe that for this placement, the hot part of the function occupies three 64-byte cache lines. Arguably a better ordering is the lower-right in Fig. 2 , which uses only two cache lines for the five hot blocks, $B_{0}, B_{1}, B_{2}, B_{3}, B_{4}$, at the cost of breaking the lightly weighted branch $B_{6} \rightarrow B_{2}$.

How do we identify the best ordering of basic blocks? The question is fairly difficult and even experts may have hard time determining which ordering leads to the maximum performance of a binary. A naive approach is to exhaustively evaluate every valid block placement and then profile the binary to collect relevant performance metrics. Obviously due to the enormous search space, this approach is infeasible for practical use. A natural improvement is to reduce the search space and experiment only with the most promising orderings. This technique, also known as iterative compilation or autotuning, is a natural task for machine learning [14], [15]. While in certain scenarios the overhead is justifiable, we found this approach impractical for our production systems due to long build and deployment times. Therefore, we use another strategy for optimization by developing a score function that is used as a proxy for estimating the quality of an ordering. The idea is to perform extensive experiments profiling an application in order to understand what aspects and features of the block placement affect the resulting performance. After this first phase, employ a machine learning technique to build an optimization model and derive a quality metric for an ordering. As a final step, design an algorithm to optimize the constructed score function.

Next we describe the process in detail, explaining the data collection phase and presenting the developed score function. Since our new approach for basic block reordering is implemented in a post-link optimizer BOLT [5] and evaluated on modern Intel x86 processors, we describe the steps that are typical for this setup. Note however that the approach is not tied to the binary optimizer and can be similarly applied in other environments. 

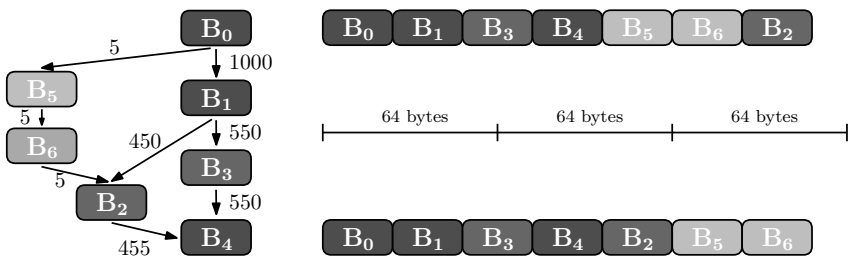

Fig. 2. A control flow graph with jump frequencies (left) and two possible orderings of basic blocks (right). All blocks have the same size (in bytes) and colored according to their hotness in the profile. An optimal TSPbased layout (upper right) utilizes three cache lines for the hot code, while an arguably better layout (lower right) can be built with a new EXTTSP model.

\subsection{Data Collection}

Following most of the recent works on profile-guided code optimizations [1], [2], [4], [5], we rely on sampling techniques for collecting profile data. Although the sampling-based approach is typically less accurate than the instrumentation-based one, it incurs significantly less memory and performance overheads, making it the preferred way of profiling binaries in actual production environments. We utilize hardware support of Intel x86 processors to collect Last Branch Records (LBR), which is a list of the last 16 taken branches. From the list of branches, that are sampled according to a specified event, we infer the frequencies of jumps between basic blocks. Specifically, we extract a weighted directed control flow graph for every function in the profiled binary. The vertices (basic blocks) and the edges (branches) of the graph, along with the sizes of the blocks, are extracted statically via the BOLT infrastructure, which is based on LLVM |16|. The weights between the basic blocks correspond to the total number of times the jumps appear in collected LBRs. We stress that before processing, we augment collected LBRs with fall-through jumps, as LBRs only contain information about taken branches; to this end, we utilize a simple algorithm similar to one described by Chen et al. [17]. Notice that we ignore indirect branches, procedure calls, and returns while constructing the control flow graph.

We have experimented with several different events to collect LBRs, including cycles, retired instructions, and taken branches, and using various levels of precise event based sampling. We observed that independently of the utilized event and processor microarchitecture, the extracted jump frequencies do not always follow the expected distribution; refer to [5], [17], [18] for concrete examples and possible explanations of the phenomenon. Hence, we use by default the cycles event to sample LBRs.

A common technique for ensuring edge weights are more realistic is solving the MINIMUM COST MAXIMUM FLOW problem on the control flow graph, which was initiated by Levin et al. [19] and later adopted by several groups [17], [18], [20], |21]. In contrast with the earlier works, our experiments with the flow-based approach did not produce performance gains in comparison with the original (possibly biased) data. A problematic example for the approach is illustrated in Fig. 3a, where the arguably most realistic adjustment is highlighted. Depending on how the

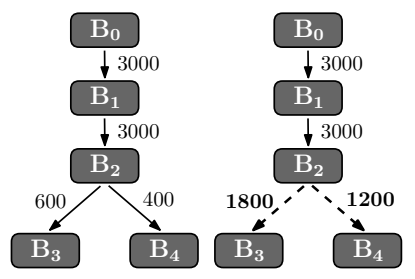

(a) (b)

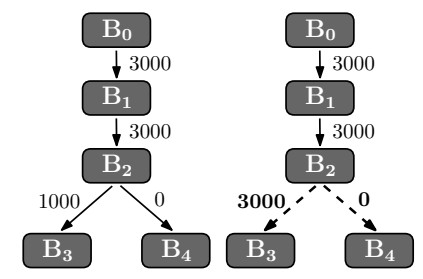

Fig. 3. Two examples of an original incomplete profile (left) and its realistic correction (right) that cannot be reconstructed using the MINIMUM COST MAXIMUM FLOW model. Bold numbers show most realistic adjustments of the edge weights satisfying flow conservation constraints.

costs of the edges are assigned, an algorithm for MINIMUM COST MAXIMUM FLOW will either produce frequencies 2600 and 400 or 600 and 2400 for jumps $B_{2} \rightarrow B_{3}$ and $B_{2} \rightarrow B_{4}$, respectively. However, it is desirable to keep the probabilities of the branches at $B_{2}$ (approximately) the same. A related issue is shown in Fig. 3b Here an algorithm may decide to send some flow along edge $B_{2} \rightarrow B_{4}$, thus making basic block $B_{4}$ hot. This adjustment prohibits future compiler optimizations that position hot and cold parts of the function in different sections of the binary. Therefore, we avoid modifying jump frequencies via the flow-based approach, leaving for the future the task of increasing profile precision.

\subsection{Engineering a Score Function}

Our goal is to design a function $x \rightarrow f(x)$, that takes in a feature vector $x$, characterizing an ordering of basic blocks, and produces a real value $f(x)$, indicating an expected performance of a binary for the ordering. We assume that the execution time of a single basic block is independent of the block ordering within a function. Thus, the ordering only affects branches between the blocks, which may incur some delay in the execution, for example due to a miss in the instruction cache. However, not all branches equally affect the performance. An important feature of a branch is the jump length, that is, the distance (in bytes) between the end of the source block to the beginning of the target block; see Fig. 4 For example, it is a common belief that zerolength jumps (equivalently, fall-through branches) impose the smallest performance overhead. This is the main motivation for the TSP model, whose objective can be formally expressed as follows:

$$
\mathrm{TSP}=\sum_{(s, t)} w(s, t) \times \begin{cases}1 & \text { if } \operatorname{len}(s, t)=0, \\ 0 & \text { if } \operatorname{len}(s, t)>0,\end{cases}
$$

where $w(s, t)$ is the frequency and len $(s, t)$ is the length of branch $s \rightarrow t$. An optimal ordering corresponds to the maximum value of the expression; thus, we call it the score of TSP. The performance, however, might also depend on other characteristics of a branch, which we discuss next. In our study, we consider the following features.

- The length of a jump impacts the performance of instruction caches. Longer jumps are more likely to result in a cache miss than shorter ones. In particular, a jump with the length shorter than 64 bytes has a chance to remain within the same cache line. 


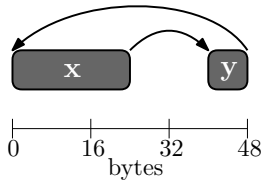

Fig. 4. The lengths of a forward jump, $x \rightarrow y$, and a backward jump, $y \rightarrow x$, are 16 and 48 bytes, respectively.

- The direction of a branch plays a role for branch predicting. A branch $s \rightarrow t$ is called forward if $s<t$, that is, block $s$ precedes block $t$ in the ordering; otherwise, the branch is called backward.

- The branches can be classified into unconditional (if the out-degree is one) and conditional (if the out-degree is two). A special kind of branches is between consecutive blocks in the ordering that are called fall-through; in this case, a jump instruction is not needed.

We introduce a new score that estimates the quality of a basic block ordering taking into account the branch characteristics. In the most generic form, the new function, called EXTENDED TSP (EXTTSP), is expressed as follows:

$$
\operatorname{ExtTSP}=\sum_{(s, t)} w(s, t) \times K_{s, t} \times h_{s, t}(\operatorname{len}(s, t)),
$$

where the sum is taken over all branches in the control flow graph. Here $w(s, t)$ is the frequency of branch $s \rightarrow t$ and $0 \leq K_{s, t} \leq 1$ is a weight coefficient modeling the relative importance of the branch for optimization. We distinguish six types of branches arising in code: conditional and unconditional versions of fall-through, forward, and backward branches. Thus, we introduce six coefficients for EXTTSP. The lengths of the jumps are accounted in the last term of the expression, which increases the importance of short jumps. A non-negative function $h_{s, t}(\operatorname{len}(s, t))$ is defined by value of 1 for zero-length jumps, value of 0 for jumps exceeding a prescribed length, and it monotonically decreases between the two values. To be consistent with the objective of TSP, the EXTTSP score needs to be maximized for the best performance. Notice that EXTTSP is a generalization of TSP, as the latter can be modeled by setting $K_{s, t}=1, h(\operatorname{len}(s, t))=1$ for fall-through branches and $K_{s, t}=0$ otherwise.

In general we cannot manually select the most appropriate constants of EXTTSP that best model the performance of modern processors. Next we describe a process for learning these constant values that lead to the best performance.

\subsection{Learning Parameters}

As a preliminary step of our study, we run multiple experiments with two binaries, the Clang compiler and the HipHop Virtual Machine (HHVM) |22|. Each experiment consists of constructing a distinct ordering of basic blocks, running a binary, and measuring its performance metrics via the Linux perf tool. In order to build a variety of block orderings for the same binary, we utilize five algorithms (described in Section 5.1) and apply them for a certain percentage of randomly selected functions. In total, we evaluated 50 distinct block orderings and conducted 250 experiments (five per ordering) for each of the two binaries.

Our first finding is that the traditional TSP score has a relatively high correlation with the performance of the binaries; see Fig. 6 However, there are several unexpected outliers that cannot be explained by the model. In order to choose suitable parameters for the ExTTSP score, we employ the so-called black-box solver developed by Letham et al. [23], which is a powerful tool for optimizing functions with computationally expensive evaluations. Formally, our problem can be stated as finding parameters for EXTTSP that have the highest correlation with the performance of a binary in the experiments. Here we try to maximize the Kendall rank correlation coefficient, $\tau$, between the observed performance (instructions per cycle) and the predicted improvement given by the EXTTSP score. Notice that the Pearson correlation coefficient, $\rho$, is not the best choice for optimization, as the relationship between observed and predicted values might not be linear. The black-box solver, which is based on Bayesian optimization, is able to compute values for a collection of continuous parameters that maximize the correlation coefficient. This is done via a careful exploration of the search space taking into account noise in real-world experiment outcomes. In our study we introduce six variables for weight coefficients, $K$, of ExtTSP. The jump-length function, $h(\cdot)$, is considered to be of the form $\left(1-\left(\frac{\operatorname{len}(j u m p)}{M}\right)^{\alpha}\right)$ with two variables $M>0$ and $\alpha>0$ for different types of branches.

The black-box solver found a model that better predicts the observed values; see Fig. 6 . The new model increases the Kendall correlation coefficient $\tau$ from 0.877 to 0.906 for Clang and from 0.897 to 0.921 for HHVM. The models constructed for Clang and HHVM are not identical, though they share many similarities. Next we present a unified variant of the model in which we round and combine parameters with similar weights (having difference less than 0.05 ), and exclude ones having small values (less than 0.05 ). We did not notice a discrepancy between the actual and the rounded EXTTSP models, meaning that the resulting solution works well for both of the binaries and is robust to the choice of constants. Recall that better block orderings correspond to higher values of EXTTSP.

$\operatorname{ExtTSP}=\sum_{(s, t)} w(s, t) \times \begin{cases}1 & \text { if } \operatorname{len}(s, t)=0 \\ 0.1 \cdot\left(1-\frac{\operatorname{len}(s, t)}{1024}\right) & \text { if } 0<\operatorname{len}(s, t) \leq 1024 \\ & \text { and } s<t \\ 0.1 \cdot\left(1-\frac{\operatorname{len}(s, t)}{640}\right) & \text { if } 0<\operatorname{len}(s, t) \leq 640 \\ & \text { and } t<s \\ 0 & \text { otherwise }\end{cases}$

Intuitively, EXTTSP resembles the traditional TSP model, as the number of fall-through branches is the dominant factor. The main difference is that EXTTSP rewards longer jumps. The impact of such jumps is significantly lower and it linearly decreases with the length of a jump. Next we summarize our high-level observations regarding the new score function. 


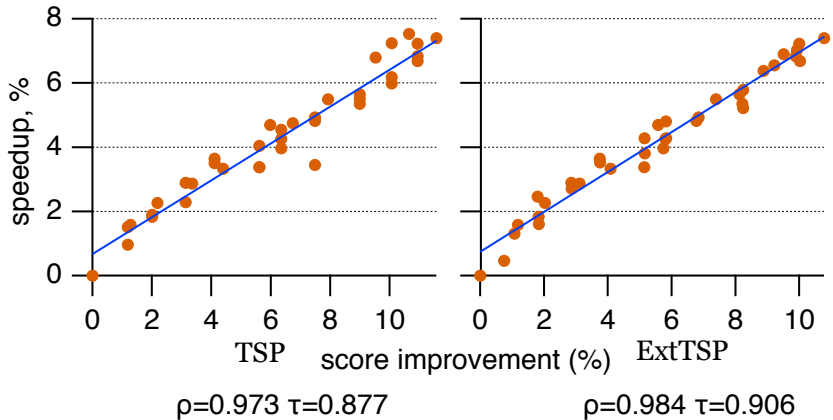

(a) Clang

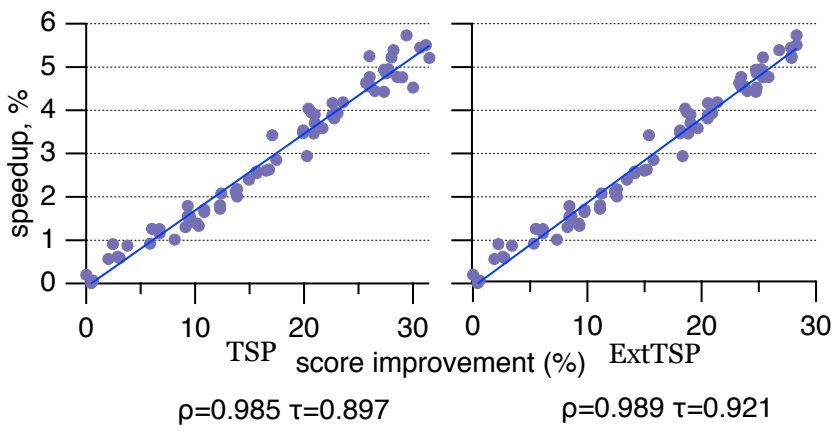

(b) HHVM

Fig. 6. The relationship between the performance (instructions per cycle) of two binaries and the TSP and ExTTSP scores measured for various orderings of basic blocks. The values correspond to the relative improvements over the non-optimized binary with the original ordering provided by the compiler.

- The suggested parameters for EXTTSP correlate well with the overall performance of a binary in a production-like environment, though we also observe moderate correlation (in the order of $\rho=0.8$ ) between the values of EXTTSP and the measured number of Icache misses.

- We have not observed significant differences between the importance of conditional and unconditional branches that seem to be similarly relevant for the quality of a block ordering. It contradicts to the intuition of Calder and Grunwald [11] who assign noticeably different weights depending on the type of a branch.

- The maximum length of a jump affecting EXTTSP is fairly large: 16 and 10 cache lines for forward and backward branches, respectively. The importance of a nonfall-through branch linearly decreases with its length; see Fig. 5 . We experimented with non-linear decreasing functions but did not discover a significant improvement; hence, we use the simpler variant.

- We found that forward branches are more important for an ordering than backward ones; see Fig. 5 for a dependency of the weights of the two types of branches on the EXTTSP score. Both types of non-fall-through branches are noticeably less important than fall-throughs in the constructed model, which is reflected in the low coefficient (0.1) in the expression.

Finding an optimal solution for the EXTENDED TSP problem is NP-hard. Next we describe our heuristic.

\section{A HeURISTIC fOR EXTTSP}

Our algorithm finds an optimized ordering of basic blocks for every function in the binary. It operates with a weighted control flow graph $G=(V, E, w)$ containing a set of basic blocks, $V$, and directed edges, $E$, representing branches between the blocks. An edge $(s, t) \in E$ corresponds to a jump from a block $s \in V$ to a block $t \in V$ and its weight, $w(s, t)$, corresponds to the frequency of the jump. We assume that the sizes (in bytes) of the basic blocks are a part of the input. The goal of the algorithm is to find an ordering of $V$ with an improved ExtTSP score (as defined in Section 2) while keeping a given entry point, $v^{*} \in V$, the first in the ordering.

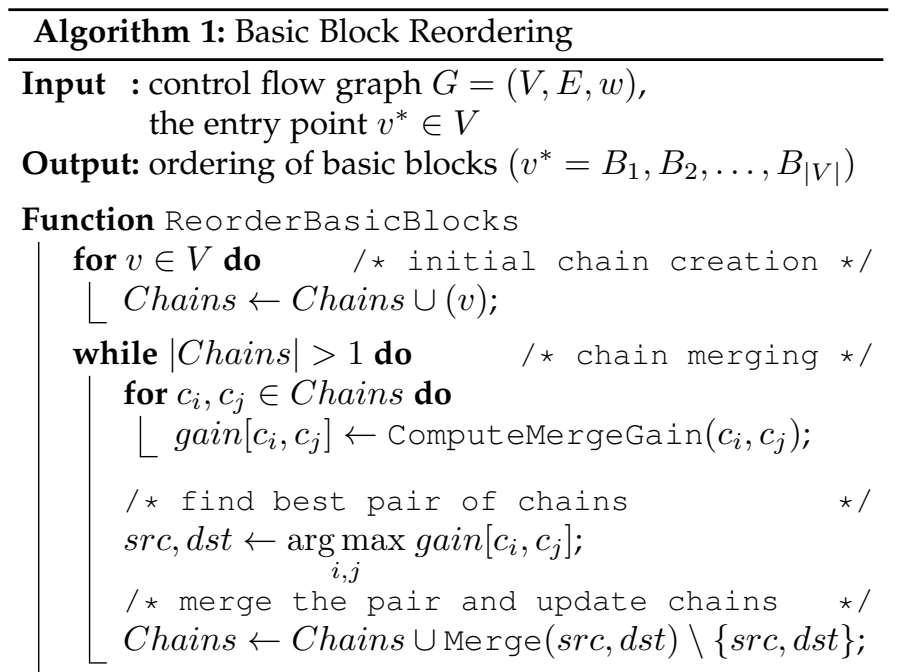

return ordering given by the remaining chain;

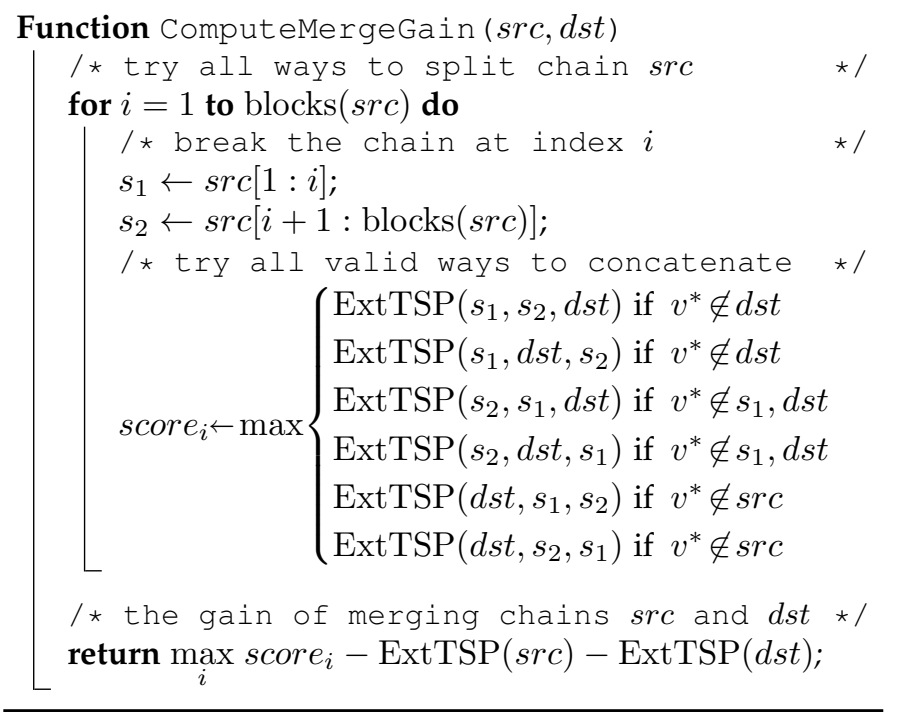

On a high level, our algorithm is a greedy heuristic that works with chains (ordered sequences) of basic blocks; see Algorithm 1 for an overview. Initially all chains are isolated basic blocks. Then we iteratively merge pairs of chains so as 
to improve the ExtTSP score. On every iteration, we pick a pair of chains whose merging yields the biggest increase in ExtTSP, and the pair is merged into a new chain. The procedure stops when there is only one chain left, which determines the resulting ordering of basic blocks.

An important aspect of our approach is the way two chains are merged; see function COMPUTEMERGEGAIN of Algorithm 1. In order to merge a pair of chains, src and $d s t$, we first split chain src into two subchains, $s_{1}$ and $s_{2}$, that retain the ordering of blocks given by src. Then we consider all six possible ways of combining the three chains, $s_{1}, s_{2}$, and $d s t$, into a single one, discarding the ones that do not place entry point $v^{*}$ at the beginning. A chain with the largest ExtTSP over all possible splitting indices of $\operatorname{src}$ and permutations of $s_{1}, s_{2}$, dst is chosen as the result. The motivation here is to increase the search space in comparison to the simpler concatenation of two chains. The simplest example in which chain splitting helps is depicted in Fig. 7 A greedy concatenation merges block $B_{0}$ with $B_{2}$ on the first iteration, which results in the final ordering $\left(B_{0}, B_{2}, B_{1}\right)$. In contrast, chain splitting allows to build an ordering $\left(B_{0}, B_{1}, B_{2}\right)$, which has a higher EXTTSP score since all the edges are forward.

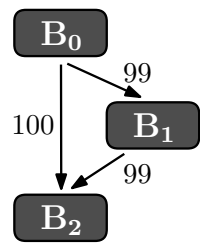

Fig. 7. An example of a control flow graph in which naive chain concatenation produces suboptimal ordering.

What is the computational complexity of Algorithm 1? A naive implementation takes $\mathcal{O}\left(|V|^{5}\right)$ steps: There are $|\vec{V}|$ merge iterations that process at most $|V|^{2}$ pairs of chains per iteration with $\mathcal{O}\left(|V|^{2}\right)$ steps needed to compute a merge gain between two chains. However, this is an overestimation, as we argue below. First observe that the ExtTSP score between two chains, $c_{1}$ and $c_{2}$, can be positive only if there is a branch between $c_{1}$ and $c_{2}$; thus, the number of candidate chain pairs for merging is upper bounded by the total number of branches in the control flow graph, $|E|$. The second observation is that one can memoize the results of COMPUTEMERGEGAIN function and re-use them throughout the computation. It is easy to see that the merge gain depends only on a pair of chains; hence, if neither of the two chains is merged at an iteration, then we do not need to recompute the gain for the pair at the next iteration. Putting the two observations together, the running time of Algorithm 1 is bounded by $\mathcal{O}\left(\sum_{c} \operatorname{blocks}(c) \cdot \operatorname{degree}(c)\right)$, where the sum is taken over all chains taking part in a merge with blocks $(c)$ being the number of blocks in the chain and degree $(c)$ being the number of branches from and to the chain. In the worst case, this sums up to $\mathcal{O}\left(|V|^{2}|E|\right)$ time in general, which equals to $\mathcal{O}\left(|V|^{3}\right)$ for real-world control flow graphs.

\subsection{Large functions}

While cubic running time is acceptable for reordering most of the functions we experimented with, there are several exceptions with a large number of basic blocks. In order to deal with these cases, we introduce a threshold, $k>1$, on the maximum size of a chain that is considered for splitting in COMPUTEMERGEGAIN. If the size of a chain, $c$, exceeds the threshold, that is, $\operatorname{blocks}(c)>k$, then we only try a simple concatenation of $c$ with other chains. With the modification, the complexity of Algorithm 1 is estimated by $\mathcal{O}\left(k \cdot|V|^{2}\right)$, which is quadratic when $k$ is a constant. In our implementation, we use $k=128$ as the default value.

\subsection{Reordering of cold blocks}

Notice that Algorithm 1 is not trying to optimize layout of cold basic blocks that are never sampled during profiling. However, one may still want to modify their relative order, as this could affect the code size as follows. Consider pairs of cold basic blocks, $s$ and $t$, such that the only outgoing branch from $s$ is the only incoming branch to $t$. If $s$ and $t$ are not consecutive in the resulting ordering, then one would need to introduce an unconditional branch instruction. In contrast, if $t$ follows $s$ in the ordering, then the instruction is not needed as $t$ is on the fall-through path of $s$. In order to guarantee that Algorithm 1 always merges such pairs into a chain, we modify the weights of cold edges in the control flow graph before the computation. Specifically we set $w(s, t)=\epsilon_{1}$ (for some $0<\epsilon_{1} \ll 1$ ) if $(s, t) \in E$ corresponds to a cold fall-through branch in the original binary, and set $w(s, t)=\epsilon_{2}$ (for some $\left.0<\epsilon_{2}<\epsilon_{1}\right)$ if $(s, t)$ corresponds to a cold non-fall-through branch. Such weights make it desirable to merge original fall-through branches, even if they are cold according to the profile.

\subsection{Code layout in memory}

Apart from basic block reordering, profile-guided optimization tools typically perform two other passes directly affecting the layout of functions in the generated code: hot/cold code splitting and code alignment [1], [5]. The first one splits hot and cold basic blocks into separate sections, while the second pass aligns the blocks at cache line boundaries via introducing NOP instructions. We stress that both optimizations are complimentary to basic block reordering and their benefits are additive. In our experiments, we evaluate the effect of reordering alone, with all other optimization passes applied for the binary.

\section{An Optimal Algorithm for ExtTSP}

We now demonstrate how EXTTSP is formulated as a Mixed Integer Program (MIP). A MIP is a method to find optimal solutions for NP-hard problems whose objective and requirements are represented by linear functions. This is a timeconsuming technique that can be applied only for small instances, and we use the optimal MIP solutions to better understand the quality of our heuristic. 
TABLE 1

maximize $\sum_{(s, t)} w(s, t) \times f\left(d_{s, t}\right)$

$$
\text { subject to } \begin{aligned}
x_{s} & \in \mathbb{R}, & & s \in V \\
d_{s, t} & \in \mathbb{R}, & & (s, t) \in E \\
z_{s, t} & \in\{0,1\}, & & s, t \in V, s \neq t \\
x_{t}-x_{s} & \geq L_{s}-M & & \\
& \times\left(1-z_{s, t}\right), & & s, t \in V, s \neq t \\
x_{s}-x_{t} & \geq L_{t}-M \cdot z_{s, t}, & & s, t \in V, s \neq t \\
d_{s, t} & =x_{t}-x_{s}-L_{s}, & & (s, t) \in E
\end{aligned}
$$

The objective is a summation over the contribution of each edge $(s, t) \in E$ in the control flow graph to EXTTSP. The contribution of an edge is the number of jumps, $w(s, t)$, weighted by a value dependent on the length of the jump, $d_{s, t}=\operatorname{len}(s, t)$. The piece-wise function shown in Fig. 5 converts the length to the desired weight. This function is formulated in MIP by introducing additional integer variables to cope with the non-convex shape [24].

The constraints express the complete search space of all legal starting bytes $x_{s}$ for each block $s \in V$ considering the size of the block, $L_{s}$. For all pairs of blocks, $s$ and $t$, either the final ordering has $s$ before $t$ (that is, $x_{t}-x_{s} \geq$ $L_{s}$ ) or $t$ before $s$ (that is, $x_{s}-x_{t} \geq L_{t}$ ). A binary variable $z_{s, t}$ is utilized to enforce one of those two constraints. The distances used in the objective, $d_{s, t}$, are constrained to be the distance between the end of block $s$ and the start of block $t$. Negative distances correspond to backward jumps which are incorporated into the piece-wise function in the objective. We utilize the Xpress solver for finding optimal solutions of the MIP model.

\section{Evaluation}

The experiments presented in this section were conducted on Linux-based servers powered by Intel microprocessors. The applications were compiled using either GCC 8.3 or Clang 7.1 with -O3 optimization level.

\subsection{Techniques}

We compare our new algorithm (referred to as ext-tsp) with the following competitors.

- original is the ordering provided by the compiler.

- tsp is the ordering constructed by the "top-down" heuristic suggested by Pettis and Hansen [6]. The algorithm starts by placing the entry basic block for a function, and then iteratively finding a successor with the heaviest edge to the last placed block. If all successors have already been selected, then one picks the block with the largest connection to the already placed blocks.

- ph is the Pettis-Hansen "bottom-up" algorithm |6]. The algorithms maintains a collection of chains of basic blocks, which correspond to paths in the control flow graph. Initially every block forms its own chain. Looking at the arcs from largest to smallest, two different chains are merged together if the arc connects the tail of one chain to the head of another. Once the merging stage is done, the chains are ordered so as to maximize the weight of backward edges to achieve the best performance of the branch predictor.
Basic properties of evaluated binaries

\begin{tabular}{lrrrrrr}
\hline & & & hot & \multicolumn{3}{c}{ blocks per function } \\
& .text (MB) & IPC & functions & p50 & p95 & max \\
\hline HHVM & 285 & 0.83 & 12,687 & 40 & 454 & 10,664 \\
Multifeed & 395 & 0.95 & 24,037 & 15 & 151 & 9,228 \\
Proxygen & 160 & 0.63 & 9,997 & 10 & 97 & 945 \\
\hline Clang & 48 & 0.82 & 7,013 & 25 & 241 & 11,218 \\
GCC & 15 & 0.76 & 10,269 & 11 & 152 & 3,354 \\
\hline
\end{tabular}

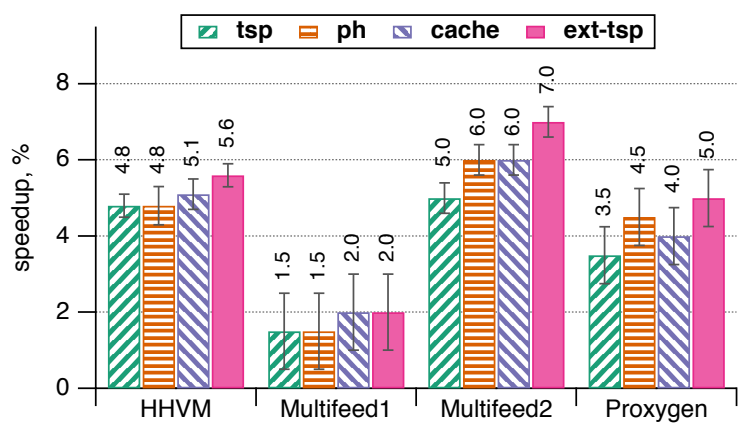

Fig. 8. Performance improvements of various reordering algorithms over original measured for different Facebook workloads.

- cache represents a modification of the Pettis-Hansen algorithm suggested by Luk et al. for the Ispike postlink optimizer [2]. The difference from $\mathrm{ph}$ is in the last step, ordering of chains of basic blocks. The chains are sorted by their density, that is, the total execution count of a chain divided by the sum of sizes of its basic blocks. Placing hottest chains first reduces conflicts in the Icache and improves code locality.

- mip is an optimal algorithm for ExtTSP described in Section 4. Since the running time of the approach is not practical for large functions, we only compare the results of mip on a subset of small functions.

All the algorithms are implemented in an open-source post-link binary optimizer BOLT [7].

\subsection{Facebook Workloads}

This section evaluates various basic block ordering algorithms on four large-scale binaries deployed at Facebook's data centers. The first system, which is our primary use case for ext-tsp, is the HipHop Virtual Machine (HHVM) [22], that serves as an execution engine for PHP at Facebook, Wikipedia, Baidu, and other large websites. The two binaries of Multifeed are responsible for News Feed. Proxygen is a Facebook service for cluster load balancing. The HHVM binary is built using GCC with LTO, while Multifeed and Proxygen are compiled with Clang with AutoFDO enabled to enhance their performance. All the Facebook services are running with huge pages enabled and utilize function reordering [4]. Table 1 provides basic properties of the evaluated binaries.

Fig. 8 presents a performance comparison of four basic block ordering algorithms on the Facebook workloads. The results are obtained by using an internal performancemeasurement tool for running A/B experiments; see [23], [25] for an overview. The tool is used at Facebook for a wide 


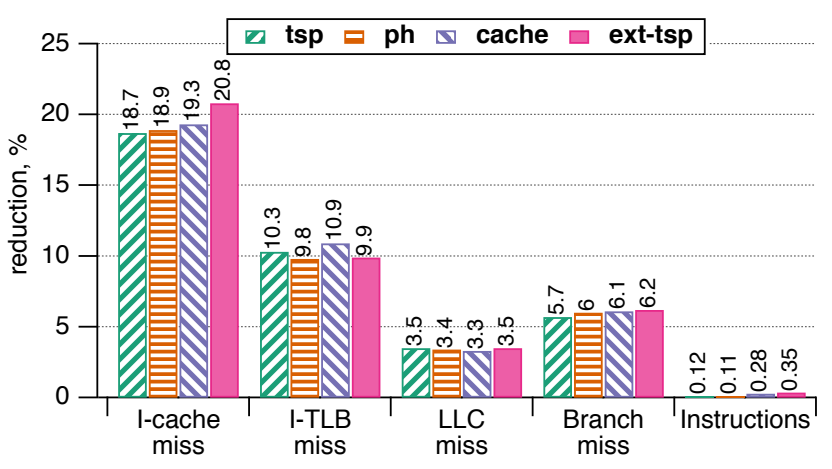

Fig. 9. perf metrics measured for the HHVM binary.

range of performance evaluations by running experiments on a set of isolated machines that process the same production traffic over several days. We measure performance as the CPU utilization during steady state. As a baseline, we observe the performance of the binaries optimized with BOLT using the original block ordering algorithm. In the case of HHVM, this is an original ordering constructed by the compiler, while for Multifeed and Proxygen, the ordering is a result of processing the binaries with PGO. The figure reports mean relative improvements of various block ordering algorithms over original along with their $95 \%$ confidence intervals. In the experiments, we notice that differences in CPU utilization among the block ordering algorithms are highly correlated with the differences in instructions per cycle (IPC).

Overall we observe that ext-tsp performs better than alternative ordering algorithms on three of the evaluated binaries. The relative speedup is close to $1 \%$ for Multifeed 2 and around $0.5 \%$ for HHVM and Proxygen. We stress that the measurements for Multifeed 1 are noticeably noisier than the alternatives with a typical deviation from the mean around $0.5 \%-1 \%$. To better understand the benefits of applying the new block ordering algorithm, we perform a more detailed evaluation of HHVM. The results are depicted in Fig. 9 The main advantage of block ordering optimization is an improved performance of the L1 I-cache, that exhibits over $19 \%$ miss reduction. The new ordering algorithm increases this value to $21 \%$. The number of branch and I-TLB misses is also significantly reduced, with ext-tsp being the best for the branch misses counter. We also see a modest improvement in the performance of the last level cache, though the difference between various ordering algorithms is not prominent.

\subsection{Open-Source Compilers}

Since basic block reordering primarily improves the performance of the I-cache, our optimization can be beneficial for any front-end bound application with large code size. We illustrate this by experimenting with binaries of two opensource compilers, Clang and GCC, whose .text sections are $48 \mathrm{MB}$ and $15 \mathrm{MB}$, respectively; see Table 1 . For these experiments, we utilize a dual-node 28 -core $2.4 \mathrm{GHz}$ Intel Xeon E5-2680 (Broadwell) with 256GB RAM. The size of the L1 I-cache on the processor is only $32 \mathrm{~KB}$, which makes the two binaries good candidates for profile-guided layout optimizations.

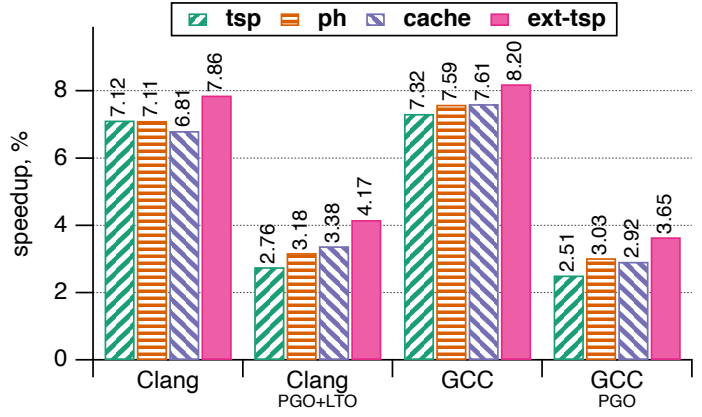

Fig. 10. Performance improvements of various reordering algorithms over original measured for Clang and GCC.
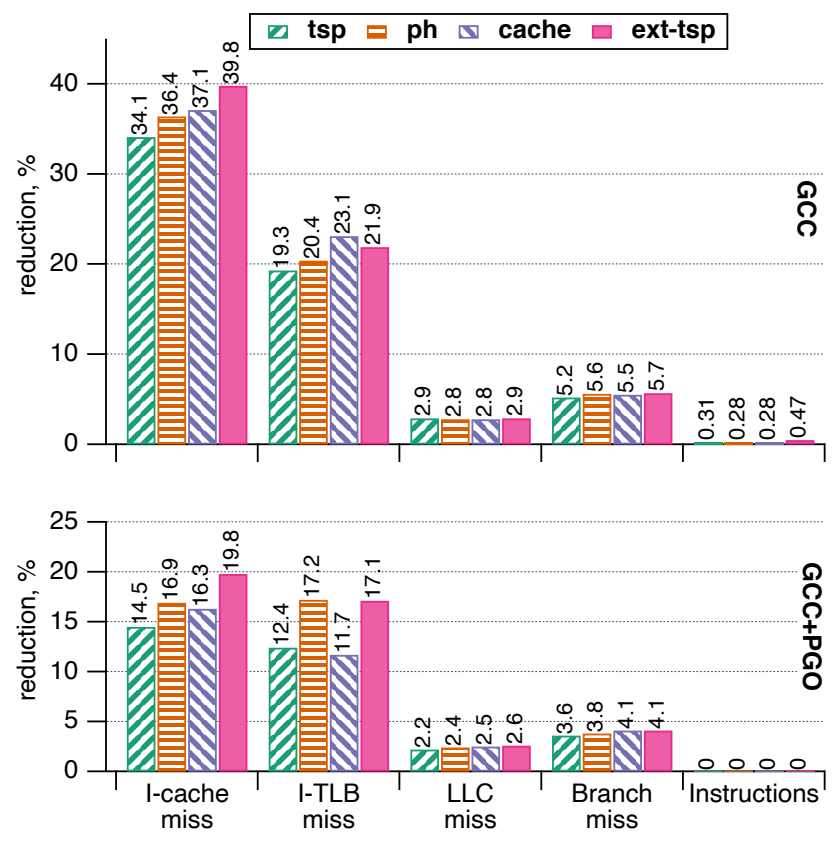

Fig. 11. perf metrics measured for the GCC binary with (bottom) and without (top) PGO applied. The improvements are on top of the original block ordering using the same configuration.

For the evaluation of Clang, we use the release_71 branch of LLVM. We ran the evaluation in two modes, Clang and Clang+PGO+LTO; see Fig. 10 First we build a release version of the binary using GCC and collect a profile data by compiling a medium-sized template-heavy $\mathrm{C}++14$ source file. For the first experiment (Clang), we optimize the binary of Clang with BOLT using various orderings of basic blocks, and compile a different collection of about $100 \mathrm{C}++14$ source files. Hence, the train and the test datasets are different in the evaluation. Every experimental run is repeated 1000 times to increase precision of our measurements so that the average mean deviation is within $0.05 \%$. The baseline in Fig. 10 corresponds to a binary processed with BOLT using the original ordering of basic blocks. Thus, the improvements are attributed only to block reordering, while all other optimizations (e.g., function reordering or inlining) are the same across the experiments.

For our next experiment, Clang+PGO+LTO, we in addition enable PGO and LTO support. To this end, we first built an instrumented version of Clang, and then used the instrumented compiler to build the binary again with the 
default options of GCC. The collected profile data was used to do another build of Clang with PGO and LTO enabled. The results in Fig. 10 indicate that block reordering alone provides $2 \%-4 \%$ performance improvements, even when applied on top of GCC with PGO and LTO. This finding is consistent with earlier evaluations of BOLT, where the gains are attributed to an improved code layout [5].

For the evaluation of GCC, we use version 8.3 of the compiler. Again, we collected a profile data by compiling the single source file, and perform testing using the larger $\mathrm{C}++14$ project. Similar to Clang, we ran experiments in two modes; however, we did not use LTO due to build errors related to $\mathrm{C}++$ exceptions. The effect of applying various block ordering techniques on two versions of the GCC binary (with and without PGO enabled) is shown in Fig. 10 As expected, the relative improvements are smaller for the binary built with PGO. The new algorithm, ext-tsp, provides the largest gains outperforming competitors by $0.5 \%-0.8 \%$; the differences are identified as statistically significant. Fig. 11 presents the impact of block ordering algorithms on key architectural metrics for GCC. As in the case with HHVM, the improvements are largely attributed to a reduction in I-cache and I-TLB misses. Other relevant metrics are also improved in comparison with the original ordering but there is little difference among the ordering techniques.

\subsection{SPEC CPU 2017}

In this section we evaluate basic block reordering on the SPEC CPU 2017 benchmark. We utilize $16 \mathrm{C} / \mathrm{C}++$ programs compiled using GCC with LTO and ran experiments on the same hardware as in the previous section. We analyze the performance of the binaries optimized by BOLT with various ordering algorithms, using original as a baseline. Profile data is collected using a separate SPEC train mode.

We observe that the SPEC binaries are much smaller than the typical applications used in modern data centers. Therefore, they are unlikely to be front-end bound and exhibit many I-cache and I-TLB misses. Fig. 12 presents the results of our experiments on the largest binaries that contain at least 100KB of hot code according to the collected profile. We do not see a consistent advantage of applying basic block reordering for the binaries. In most of the experiments we record a high variance in the running times, which often exceeds the differences between means. An optimized block ordering yields a statistically significant improvement over original only for three binaries: gcc 4.5 and namd (using ext-tsp), and xalancbmk (using cache). For the largest program, gcc 4.5 , the ext-tsp algorithm achieves $1.5 \%$ speedup outperforming the best competitor by $0.4 \%$.

To understand the source of regressions, we analyze two binaries from the benchmark, $\times 264$ and omnetpp, whose running times increase by $0.5 \%-1.5 \%$ after block reordering. In the former case, we observe a substantial growth in the number of branch misses, which leads to the performance regression. In the latter case, we found that a different alignment of hot loops in the binary worsen the performance despite a significant increase (20\%) of the number of fall-through branches and a modest improvement (around 5\%) in the number of I-cache misses. We conclude that for small binaries that are not front-end bound, both TSP and EXTTSP are not accurate models.

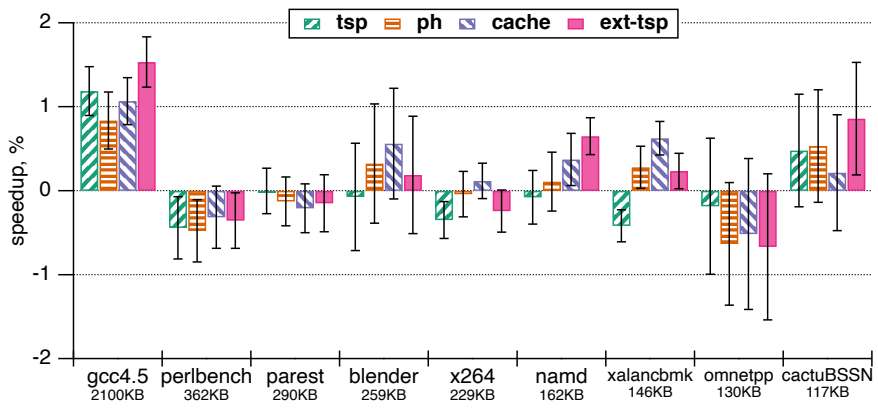

Fig. 12. Relative performance differences between original and alternative block reordering methods on the largest binaries of the SPEC 2017 dataset. Positive values indicate improvements, negative ones indicate regressions. For every binary, the size of the hot code is specified.

\subsection{Analysis of ExTTSP}

Here we present an evaluation of Algorithm 1 for solving the EXTTSP problem. We design the experiments to answer two questions: (a) How do various parameters of the algorithm contribute to the solution and what are the best default values? (b) How does the algorithm perform in comparison with existing heuristics and the optimal technique?

Considering the first question, we observe that ext-tsp has only one parameter that affects its quality and performance. As explained in Section 3, we introduce a threshold $k$, which controls the maximum size of a chain that can be split during optimization. In the extreme case with $k=|V|$, all chains can be broken if that improves the objective; however, the running time of the algorithm is cubic on the number of basic blocks comprising a function. Another extreme, $k=0$, forbids chain splitting but makes the running time quadratic. As Table 1 illustrates, some functions in the dataset contain a few thousand of basic blocks. Hence, the threshold should be chosen carefully, since it impacts the quality of a solution and the time needed to process a binary, which is important in production environments.

Fig. 13 illustrates the results of the experiments with the chain splitting threshold. Multifeed 1 is the binary whose processing time is substantially affected by large values of $k$. For $k \geq 1024$, the combined running time of ext-tsp on all functions of the Multifeed1 binary is around 2 minutes, while for HHVM it is less than 20 seconds. For $k=0$, the processing times are 6 and 4 seconds for the binaries, respectively. The difference between the corresponding solutions in the EXTTSP score ranges between $0.3 \%$ and $0.7 \%$; note that according to our analysis in Section 2 and Fig. 6. this translates to a performance difference of $0.1 \%-0.3 \%$. The value $k=128$ provides a reasonable compromise between processing speed and solution quality, and thus, it is utilized as the default value for ext-tsp in all our experiments.

In order to analyze the quality of solutions for EXTTSP generated by Algorithm 1. we employ the optimal technique presented in Section 4 We apply mip to all 2992 functions containing at most 30 basic blocks in the HHVM binary. For these small functions, mip finds a provably optimal solution in $2963(99 \%)$ of the cases in less than one minute. Out of these instances with the known optimal ordering, ext-tsp finds an equivalent solution in $2914(98.3 \%)$ cases. For the remaining 49 functions, the EXTTSP score produced by 

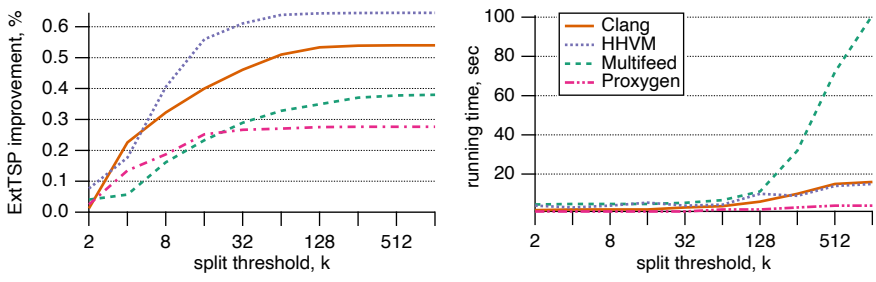

Fig. 13. The running times and the resulting EXTTSP scores produced by ext-tsp (Algorithm 1 using various chain splitting thresholds for binaries described in Table 1

ext-tsp is on average $0.14 \%$ lower than the optimum. For comparison, the runner-up approach on the same binary is cache, which is able to reconstruct 2745 (92.6\%) of optimal orderings in the binary. The relative improvements in the EXTTSP score over non-reordered functions are $27 \%, 29 \%$, $29 \%, 31 \%$ for $\mathrm{tsp}, \mathrm{ph}$, cache, and ext-tsp, respectively, which aligns with our experiments illustrated in Fig. 8

We emphasize that mip is not considered a practical approach, as it does not scale to instances with many basic blocks. The average running time of mip on a function with 30 blocks exceeds 10 seconds, while it is below a millisecond for all four alternatives, $t s p, p h$, cache, and ext-tsp. Nevertheless, the aforementioned analysis demonstrates that the new heuristic provides a close-tooptimal solutions in the majority of real-world instances, while being sufficiently fast to process large functions.

\section{Related Work}

There exists a rich literature on profile-guided optimizations. Here we discuss previous works that are closely related to code layout and our main contributions.

The work by Pettis and Hansen $|\overline{6}|$ is the basis for the majority of modern code reordering techniques. The goal is to create chains of basic blocks that are frequently executed together in the order. As discussed in Section 2 many variants of the technique have been suggested in the literature and implemented in various tools [2], [3], [8], [9], [10], [11], [12], [13]. Similar to our work, the techniques are operating with a control flow graph and try to lay out basic blocks tackling a variant of the TRAVELING SAleSMAN PROBlem. Alternative models have been studied by Bahar et al. |26], Gloy et al. [27], and Lavaee and Ding |28|, where a temporal-relation graph is taken into account. Temporal affinities between code instructions can be utilized for reducing conflict cache misses [29] and improving the performance of multiple applications using a shared cache $|30|$. We emphasize that according to our experiments, the performance of a front-end bound largescale binary can be largely predicted by its control flow graph without considering more expensive models.

Code reordering at the function-level is also initiated by Pettis and Hansen [6], who describe an algorithm that is implemented in many compilers and binary optimization tools [5], [13], [31], [32]. This approach greedily merges chains of functions and is designed to primarily reduce ITLB misses. An improvement is recently proposed by Ottoni and Maher [4], who suggest to work with a directed call graph. Note that unlike our work, the techniques are heuristics not aiming to produce code layouts that are optimal from the performance point of view.

Another opportunity for improving performance is to modify layout of data [33], [34], [35], [36]. Most of the existing works focus on field reordering and structure splitting based on the field hotness and data affinities. While the problem of finding an optimal data layout is computationally hard [37], |38], we believe that utilizing machine learning may lead to improved heuristics resulting in performance gains for real-world applications.

\section{CONCLUSION}

In this work we extended the state-of-the-art model for reordering of basic blocks and developed a new efficient algorithm to optimize the layout of a binary. We also performed an extensive evaluation of various block ordering techniques on a variety of real-world applications. The experiments indicate that the new technique can improve the performance of binaries that have been manually tuned over the course of their development and optimized using conventional compiler optimizations. There are several interesting aspects of our approach that we discuss next.

Firstly, our approach employs a machine learning toolkit to build a desired objective for optimization. As our evaluation demonstrates, the resulting model outperforms the classical one, as the new objective correlates very well with the performance of large-scale binaries. A possible risk here is to over-tune a model for a specific application and miss important details that might affect performance. The experiments with the SPEC benchmark imply that the models based on maximizing the number of fall-through branches are too simplistic for binaries that are not front-end bound.

Secondly, our study focuses on optimizing applications built with particular compilers and running on a specific hardware. A reasonable future work is to verify whether the presented approach can be generalized to other use cases. Our preliminary experiments indicate that comparable gains can be achieved on other Intel microprocessors and alternative processor architectures. Similarly, the new reordering algorithm is applied as a post-link optimization, and we did not examine how it behaves on earlier compilation stages. It would be interesting to investigate the effect of the reordering applied at compilation time. In particular, we plan in the future to integrate and compare ext-tsp with the algorithms implemented in GCC [12] and Clang.

Finally, we point out that this paper considers a certain aspect related to code generation: reordering of basic blocks within a function. There are many complementary optimizations that we did not investigate in detail, for example, unrolling loops or duplicating blocks in order to avoid extra jumps. An attractive direction is to allow cross-procedure reordering in which basic blocks from different functions can be interleaved in the final layout. This might further increase code locality and improve cache utilization. Unfortunately, our preliminary experiments with existing cross-procedure heuristics [12] did not produce measurable gains; further research of the technique is an intriguing future work. 


\section{ACKNOWLEDGMENTS}

We thank Alon Shalita for fruitful initial discussions of the project. We would also like to thank Rafael Auler and Maksim Panchenko for their help with integrating the new technique into BOLT.

\section{REFERENCES}

[1] D. Chen, D. X. Li, and T. Moseley, "AutoFDO: Automatic feedback-directed optimization for warehouse-scale applications," in International Symposium on Code Generation and Optimization. ACM, 2016, pp. 12-23.

[2] C.-K. Luk, R. Muth, H. Patil, R. Cohn, and G. Lowney, "Ispike: A post-link optimizer for the Intel $($ Itanium $(R$ architecture," in Code Generation and Optimization: Feedback-Directed and Runtime Optimization. IEEE Computer Society, 2004, p. 15.

[3] B. Schwarz, S. Debray, G. Andrews, and M. Legendre, "PLTO: A link-time optimizer for the Intel IA-32 architecture," in Workshop on Binary Rewriting, 2001, pp. 1-7.

[4] G. Ottoni and B. Maher, "Optimizing function placement for largescale data-center applications," in International Symposium on Code Generation and Optimization. IEEE Press, 2017, pp. 233-244.

[5] M. Panchenko, R. Auler, B. Nell, and G. Ottoni, "BOLT: A practical binary optimizer for data centers and beyond," in International Symposium on Code Generation and Optimization. IEEE Press, 2019, pp. 2-14.

[6] K. Pettis and R. C. Hansen, "Profile guided code positioning," in SIGPLAN Notices, vol. 25, no. 6. ACM, 1990, pp. 16-27.

[7] "Binary Optimization and Layout Tool," https://github.com/ facebookincubator/BOLT

[8] F. T. Boesch and J. F. Gimpel, "Covering points of a digraph with point-disjoint paths and its application to code optimization," Journal of the ACM, vol. 24, no. 2, pp. 192-198, 1977.

[9] C. Young, D. S. Johnson, M. D. Smith, and D. R. Karger, "Nearoptimal intraprocedural branch alignment," SIGPLAN Notices, vol. 32, no. 5, pp. 183-193, 1997.

[10] T. Hirata, A. Maruoka, and M. Kimura, "A polynomial time algorithm to find a path cover of a reducible flow graph," Syst. Comput. Control, vol. 10, no. 3, pp. 71-78, 1979.

[11] B. Calder and D. Grunwald, "Reducing branch costs via branch alignment," in SIGPLAN Notices, vol. 29, no. 11. ACM, 1994, pp. 242-251.

[12] J. Torrellas, C. Xia, and R. L. Daigle, "Optimizing the instruction cache performance of the operating system," IEEE Transactions on Computers, vol. 47, no. 12, pp. 1363-1381, 1998.

[13] A. Ramírez, J.-L. Larriba-Pey, C. Navarro, J. Torrellas, and M. Valero, "Software trace cache," in International Conference on Supercomputing. ACM, 2014, pp. 261-268.

[14] A. H. Ashouri, W. Killian, G. P. John Cavazos, and C. Silvano, "A survey on compiler autotuning using machine learning," $A C M$ Comput. Surv., vol. 51, no. 5, pp. 96:1-96:42, 2018.

[15] Z. Wang and M. O'Boyle, "Machine learning in compiler optimization," Proceedings of the IEEE, vol. PP, no. 99, pp. 1-23, 2018.

[16] C. Lattner and V. Adve, "LLVM: A compilation framework for lifelong program analysis \& transformation," in International Symposium on Code Generation and Optimization. IEEE Computer Society, 2004, p. 75.

[17] D. Chen, N. Vachharajani, R. Hundt, X. Li, S. Eranian, W. Chen, and W. Zheng, "Taming hardware event samples for precise and versatile feedback directed optimizations," IEEE Transactions on Computers, vol. 62, no. 2, pp. 376-389, 2013.

[18] A. Nowak, A. Yasin, A. Mendelson, and W. Zwaenepoel, "Establishing a base of trust with performance counters for enterprise workloads," in USENIX Annual Technical Conference, 2015, pp. 541548 .
[19] R. Levin, I. Newman, and G. Haber, "Complementing missing and inaccurate profiling using a minimum cost circulation algorithm," in International Conference on High-Performance Embedded Architectures and Compilers. Springer, 2008, pp. 291-304.

[20] D. Novillo, "SamplePGO: the power of profile guided optimizations without the usability burden," in Proceedings of the 2014 LLVM Compiler Infrastructure in HPC. IEEE Press, 2014, pp. 22-28.

[21] X.-H. Liu, Y. Peng, and J.-Y. Zhang, "A sample profile-based optimization method with better precision," in International Conference on Artificial Intelligence and Computer Science. DEStech, 2016, pp. 340-346.

[22] K. Adams, J. Evans, B. Maher, G. Ottoni, A. Paroski, B. Simmers, E. Smith, and O. Yamauchi, "The HipHop Virtual Machine," in SIGPLAN Notices, vol. 49, no. 10. ACM, 2014, pp. 777-790.

[23] B. Letham, B. Karrer, G. Ottoni, and E. Bakshy, "Constrained Bayesian optimization with noisy experiments," Bayesian Analysis, vol. 14, no. 2, pp. 495-519, 2019.

[24] K. L. Croxton, B. Gendron, and T. L. Magnanti, "A comparison of mixed-integer programming models for nonconvex piecewise linear cost minimization problems," Management Science, vol. 49, no. 9 , pp. $1268-1273,2003$.

[25] E. Bakshy and E. Frachtenberg, "Design and analysis of benchmarking experiments for distributed internet services," in International Conference on World Wide Web, 2015, pp. 108-118.

[26] I. Bahar, B. Calder, and D. Grunwald, "A comparison of software code reordering and victim buffers," ACM SIGARCH Computer Architecture News, vol. 27, no. 1, pp. 51-54, 1999.

[27] N. Gloy and M. D. Smith, "Procedure placement using temporalordering information," Transactions on Programming Languages and Systems, vol. 21, no. 5, pp. 977-1027, 1999.

[28] R. Lavaee and C. Ding, "ABC Optimizer: Affinity based code layout optimization," University of Rochester, Tech. Rep., 2014.

[29] A. H. Hashemi, D. R. Kaeli, and B. Calder, "Efficient procedure mapping using cache line coloring," in SIGPLAN Notices, vol. 32, no. 5. ACM, 1997, pp. 171-182.

[30] P. Li, H. Luo, C. Ding, Z. Hu, and H. Ye, "Code layout optimization for defensiveness and politeness in shared cache," in International Conference on Parallel Processing. IEEE, 2014, pp. 151-161.

[31] A. Ramirez, L. A. Barroso, K. Gharachorloo, R. Cohn, J. LarribaPey, P. G. Lowney, and M. Valero, "Code layout optimizations for transaction processing workloads," in SIGARCH Computer Architecture News, vol. 29, no. 2. ACM, 2001, pp. 155-164.

[32] W. J. Schmidt, R. R. Roediger, C. S. Mestad, B. Mendelson, I. ShavitLottem, and V. Bortnikov-Sitnitsky, "Profile-directed restructuring of operating system code," IBM Systems Journal, vol. 37, no. 2, pp. 270-297, 1998.

[33] E. Raman, R. Hundt, and S. Mannarswamy, "Structure layout optimization for multithreaded programs," in International Symposium on Code Generation and Optimization. IEEE, 2007, pp. 271-282.

[34] T. Eimouri, K. B. Kent, A. Micic, and K. Taylor, "Using field access frequency to optimize layout of objects in the JVM," in Annual Symposium on Applied Computing. ACM, 2016, pp. 1815-1818.

[35] P. Roy and X. Liu, "StructSlim: A lightweight profiler to guide structure splitting," in International Symposium on Code Generation and Optimization. ACM, 2016, pp. 36-46.

[36] T. M. Chilimbi and R. Shaham, "Cache-conscious coallocation of hot data streams," in SIGPLAN Notices, vol. 41, no. 6. ACM, 2006, pp. 252-262.

[37] E. Petrank and D. Rawitz, "The hardness of cache conscious data placement," in SIGPLAN Notices, vol. 37, no. 1. ACM, 2002, pp. $101-112$.

[38] R. Lavaee, "The hardness of data packing," SIGPLAN Notices, vol. 51, no. 1, pp. 232-242, 2016. 